\title{
Embedding approach to the isolated adsorbate
}

\author{
M. I. Trioni and G. P. Brivio \\ Istituto Nazionale di Fisica della Materia-Dipartimento di Fisica, Università di Milano, Via Celoria 16, 20133 Milano, Italy \\ S. Crampin \\ School of Physics, University of Bath, Bath BA2 7AY, United Kingdom \\ J. E. Inglesfield* \\ Institute for Theoretical Physics, Catholic University of Nijmegen, Toernooiveld, 6525 ED Nijmegen, The Netherlands
}

(Received 22 June 1995)

\begin{abstract}
An embedding method is proposed, based upon Green-function matching, for calculating the electronic properties of an isolated adsorbate. The self-consistent single-particle Schrödinger equation is solved in a localized region containing the adsorbate and that part of the substrate mainly perturbed by it. The extended substrate is taken into account exactly by an effective embedding potential. The advantages of the method for the adsorption problem are discussed and tested by a calculation of the electronic properties of isolated $\mathrm{Si}$ and $\mathrm{N}$ adatoms on $\mathrm{Al}$, modeled as jellium. In the former case excellent agreement is found with the results previously computed by other methods, in the latter, not previously investigated by a first-principles approach, the ioniclike character of the bond is seen in the calculated charge densities and densities of states. Finally the problem of the lack of screening due to the presence of an adatom on a simple metal surface is estimated by the generalized phase-shift theory. This effect turns out to be an important contribution to the atom-surface interaction energies, and it is corrected to first order by the use of the grand-canonical energy functional.
\end{abstract}

\section{INTRODUCTION}

A microscopic understanding of the dynamics of adsorption/desorption phenomena at the gas-solid interface is extremely important, as these are the basic processes of chemical reactions at surfaces. Any dynamical calculation of adsorption requires, as input, the knowledge of the adiabatic electronic properties of the particle-surface interaction, in particular, potential energy surfaces. As dynamical processes are very sensitive to the detailed structure of the potential energy surfaces, $a b$ initio studies on the statics of adsorption have developed into a major field in surface physics. ${ }^{1,2}$

The first calculations of the adiabatic adsorption properties of atoms on simple metals date from the works of Grimley, ${ }^{3,4}$ Gunnarsson, Hjelmberg, and Lundqvist, ${ }^{5,6}$ and Lang and Williams. ${ }^{7}$ Although representing different treatments, these works share a common approach-the physical space is split up into two regions. The former, of relatively small volume, where it is assumed that chemisorption is localized, is often called the embedded region. The latter, the electronic properties of which are calculated in a lower approximation, represents the substrate. In the embedded region a Dyson equation is solved self-consistently, with the effect of the solid entering in terms of an unperturbed Green function. Simple models for the substrate have been used in these papers: jellium, in the case of Refs. 5,7, possibly also including a first order perturbation due to the lattice structure, and cubium for the work reported in Ref. 4.

More recently, it has become possible to perform more elaborate and sophisticated calculations of adsorbate systems on realistic surfaces than was possible in the pioneering studies, obtaining $a b$ initio solutions within density functional theory, ${ }^{8,9}$ using either the local density approximation
(LDA), ${ }^{10}$ or, more recently, gradient-corrected density functionals. ${ }^{11}$ The techniques used in present day studies divide naturally into different classes. Cluster methods employ a small number of atoms to represent the adsorbate and surrounding substrate. ${ }^{12}$ Another approach involves the solution of the isolated adsorbate problem on an extended substrate via the Dyson equation, but by more sophisticated methods than in the early studies. Alternatively, in the supercell approach, the simplicity afforded by three-dimensional translational periodicity is fully exploited, e.g., Refs. 13,14. Instead of an isolated adsorbate, a regular array of adsorbates is considered, periodically repeated across the surface. Also, instead of an infinitely extended substrate, the solid is represented by a thin film, upwards of three layers thick, but normally a relatively modest number. The thin film and adsorbate overlayer is repeated periodically in a direction normal to the surface, creating a fully three-dimensional periodic system, which may then be tackled by the conventional machinery of bulk electronic structure theory. The supercell technique has found favor, because of its numerical simplicity, yet an ab initio description of an isolated chemical species interacting with a semi-infinite solid can still provide more accurate information on the processes leading to adsorption. This is because it avoids adsorbate-adsorbate interactions and also takes into account the continuum electronic states of the substrate.

In this paper, we develop a different approach to the study of an isolated adsorbate on an extended substrate. ${ }^{15,16}$ Recent consideration of this problem has led to various formulations based upon the Dyson equation. These include the Greenfunction operator method due to Scheffler and co-workers, ${ }^{1718}$ where the substrate Green function is expanded in an atom-centered Gaussian orbital basis; methods 
due to Lang et al. ${ }^{19}$ and Aldén et al., ${ }^{20}$ which use atomcentered partial wave basis sets and the atomic sphere approximation; and a matrix Green-function scattering method by Feibelman. ${ }^{21}$ A rather different approach not based upon the Dyson equation, which uses analytic continuation of the spatial coordinates to complex values, has been developed by Nordlander and Tully. ${ }^{22}$ Although ours is a method for determining the Green function, it is not based upon the Dyson equation. Instead, we set up an effective Schrödinger equation within a localized region (the embedded region) containing the adsorbate and the associated perturbed region of the substrate. The influence of the extended substrate enters in the form of a nonlocal energy dependent potential, added to the Hamiltonian, which acts upon the surface enclosing the embedded volume. We determine this embedding potential from the Green function of the substrate in the absence of the adsorbate. Eventually, all the relevant physical quantities are obtained as in other methods by projecting our equation onto a suitable basis set. However, unlike methods based upon the Dyson equation, our boundary conditions do not enter as an expansion throughout the embedded volume, giving us more flexibility for the choice of the basis set. In addition, our method is based upon a variational solution of the Schrödinger equation, without any a priori biasing in the behavior of the Green function. For these reasons, we believe that our embedding method should be a useful addition to the repertoire of tools available for the study of adsorption.

In the following section, we present a derivation of the main equations of our method, and we discuss their solution. In Sec. III, we discuss the calculation of physical quantities of interest. Section IV illustrates the application of the method to adsorbates on the surface of Al modeled within the jellium approximation. This system represents a standard testing ground for different techniques for studying adsorption, and enables the accuracy and utility of our method to be assessed. Section V is devoted to conclusions.

\section{EMBEDDING}

\section{A. Outline}

The embedding method ${ }^{23-28}$ has been developed for the study of extended systems, where a localized perturbation has lowered the symmetry and has caused a significant enhancement of the complexity. There are many examples of this situation: impurities within a bulk crystal, interfaces, in general, and surfaces, in particular, adsorbates at surfaces and so on.

Embedding exploits the fact that very often in these systems the electronic charge density is significantly perturbed only within a limited region. The charge density is the central quantity in density functional theory, ${ }^{8,9}$ which is the general framework adopted here, and from it, all ground state properties may be determined. In the present context, this means quantities of interest, such as the adsorption energy, the bonding site and geometry of the adsorbate, and the potential energy surfaces for surface processes such as dissociation or diffusion. ${ }^{1}$

Since the disturbance is localized, it makes sense to see whether one can obtain the perturbed charge density from a calculation that only considers this limited region. Such an approach concentrates effort and resources on the region where the important physics is going on. The difficulty is, of course, that there is coupling to the extended system, and that this coupling is important - for example, broadening localized levels into resonances, and providing a source of electrons, which can then freely flow into and out of the perturbed region.

The extended system may be taken into account if the limited region is considered with the appropriate boundary condition. This boundary condition will influence the solution of the Schrödinger equation found within the smaller region. Since the region beyond the smaller volume remains unperturbed, the boundary condition will not depend upon the perturbation. In the embedding method, the boundary condition is implemented via a non-local, energy-dependent potential, which acts upon the dividing surface of the two regions. The embedding potential is derived from a calculation performed on the unperturbed system.

We briefly summarize the derivation of the embedding equations, highlighting pertinent points. Further detail and discussion are to be found in Refs. 23,24. The total space is partitioned into regions $I$ and $I I$ (Fig. 1). The former is the volume to be embedded, the region which contains the adsorbate and that part of the system perturbed by its presence. Region $I I$ is the rest of the extended system, containing the substrate.

A variational solution to the single-particle Schrödinger equation may be found, which explicitly depends only upon the wave function in region $I$, the region of interest. To do this, we construct a trial wave function $\phi(\mathbf{r})$, which is to be varied within region $I$ and which in region $I I$ is a solution $\psi(\mathbf{r})$ of the Schrödinger equation for the unperturbed system at energy $\varepsilon$. On the surface $S$, which divides the two volumes $I$ and $I I$, the trial wave function is continuous, $\phi\left(\mathbf{r}_{S}\right)=\psi\left(\mathbf{r}_{S}\right)$, as it must be to be a valid wave function, but a discontinuity in derivative is permitted.

The expectation value of the Hamiltonian in the whole space is given by

$$
E=\frac{\int_{I} \mathbf{d}^{3} \mathbf{r} \phi^{*} H \phi+\varepsilon \int_{I I} \mathbf{d}^{3} \mathbf{r}|\psi|^{2}+\frac{1}{2} \int_{S} \mathbf{d}^{2} \mathbf{r}_{S} \phi^{*}\left(\frac{\partial \phi}{\partial n_{S}}-\frac{\partial \psi}{\partial n_{S}}\right)}{\int_{I} \mathbf{d}^{3} \mathbf{r}|\phi|^{2}+\int_{I I} \mathbf{d}^{3} \mathbf{r}|\psi|^{2}},
$$

where $n_{S}$ is the unit vector normal to the infinitesimal surface elements $\mathbf{d}^{2} \mathbf{r}_{S}$ pointing out of the region $I$, and the surface integral term is the kinetic energy contribution arising from the discontinuity of the wave function derivative across $S$. (We use here and throughout the paper, unless otherwise stated, atomic units: $e=\hbar=m_{e}=1$.) The volume integral in region $I$ may be 
eliminated by introducing the Green function $G_{0}$ for the unperturbed system, which satisfies a zero normal-derivative boundary condition on $S$ :

$$
\frac{\partial G_{0}\left(\mathbf{r}_{S}, \mathbf{r}^{\prime} ; \varepsilon\right)}{\partial n_{S}}=0 .
$$

The surface inverse of this Green function is a generalized logarithmic derivative, which relates the amplitude and derivative of the wave function on surface $S$ :

$$
\frac{\partial}{\partial n_{S}} \psi\left(\mathbf{r}_{S}\right)=-2 \int_{S} \mathbf{d}^{2} \mathbf{r}_{S}^{\prime} G_{0}^{-1}\left(\mathbf{r}_{S}, \mathbf{r}_{S}^{\prime} ; \varepsilon\right) \psi\left(\mathbf{r}_{S}^{\prime}\right)
$$

Following Ref. 23, we can thus obtain the expectation value of the Hamiltonian with our trial function, purely in terms of quantities evaluated within or on the surface of region $I$ :

$$
E=\frac{\left[\int_{I} \mathbf{d}^{3} \mathbf{r} \phi^{*} H \phi+\frac{1}{2} \int_{S} \mathbf{d}^{2} \mathbf{r}_{S} \phi^{*} \frac{\partial \phi}{\partial n_{S}}+\int_{S} \mathbf{d}^{2} \mathbf{r}_{S} \int_{S} \mathbf{d}^{2} \mathbf{r}_{S}^{\prime} \phi^{*}\left(G_{0}^{-1}(\varepsilon)-\left.\varepsilon \frac{\partial G_{0}^{-1}(\varepsilon)}{\partial \boldsymbol{\epsilon}}\right|_{E}\right) \phi\right]}{\left[\int_{I} \mathbf{d}^{3} \mathbf{r}|\phi|^{2}-\left.\int_{S} \mathbf{d}^{2} \mathbf{r}_{S} \int_{S} \mathbf{d}^{2} \mathbf{r}_{S}^{\prime} \phi^{*} \frac{\partial G_{0}^{-1}(\varepsilon)}{\partial \varepsilon}\right|_{E} \phi\right]} .
$$

If this equation is minimized with respect to the trial function $\phi$, we obtain the following Schrödinger equation:

$$
\left(H+\frac{1}{2} \delta\left(\mathbf{r}-\mathbf{r}_{S}\right) \frac{\partial}{\partial n_{S}}\right) \phi(\mathbf{r})+\delta\left(\mathbf{r}-\mathbf{r}_{S}\right) \int_{S} \mathbf{d}^{2} \mathbf{r}_{S}^{\prime}\left(G_{0}^{-1}\left(\mathbf{r}_{S}, \mathbf{r}_{S}^{\prime} ; \varepsilon\right)+(E-\varepsilon) \frac{\partial G_{0}^{-1}\left(\mathbf{r}_{S}, \mathbf{r}_{S}^{\prime} ; \varepsilon\right)}{\partial E}\right) \phi\left(\mathbf{r}_{S}^{\prime}\right)=E \phi(\mathbf{r}) \quad \text { with } \mathbf{r} \in I .
$$

Considering each term in turn, $H$ is the Hamiltonian of the system, a sum of kinetic energy, and potential energy operators (described in more detail below). The normal derivative term on the embedding surface provides Hermiticity within region $I . G_{0}^{-1}$ is the embedding potential, constraining the trial function $\phi$ to correctly match on to the substrate wave functions $\psi$. The energy-derivative term provides a first order correction to $G_{0}^{-1}$, so that the constraint is evaluated at the working energy $E$. The correction vanishes if $G_{0}^{-1}$ is evaluated at energy $E$, as is done in practice.

\section{B. Matrix representation}

In the previous section, the trial wave function, which minimized the expectation value of the Hamiltonian, was

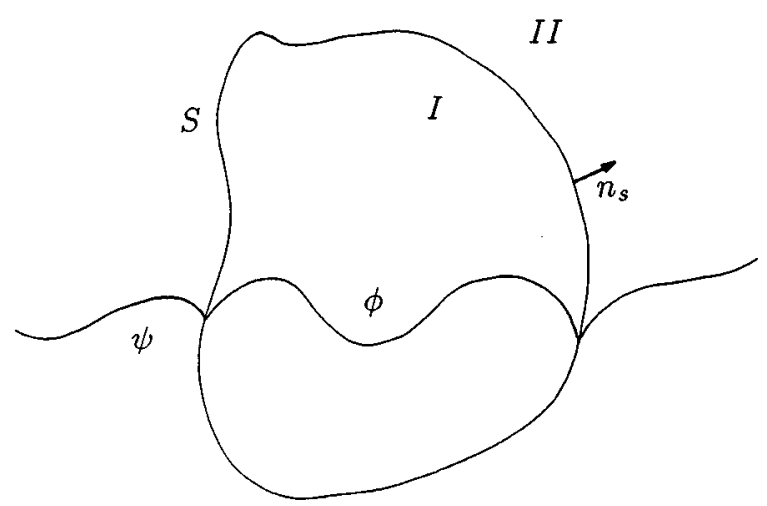

FIG. 1. The embedding geometry, with region $I$ to be embedded in the extended region $I I$. shown to satisfy an effective Schrödinger equation. To achieve this minimization in practice, we expand the trial wave function in a basis and minimize Eq. (4) with respect to the expansion coefficients, obtaining a matrix-equation representation of the Schrödinger equation.

There are beneficial reasons for switching from the electronic wave function to the single-particle Green function. The analyticity of the Green function may be exploited to simplify valence integration through the use of complex energies, and a better description is obtained of spectral features and the local density of states. Furthermore, the presence of the energy-dependent embedding potential in Eq. (5) prevents the eigenvalues and eigenvectors from being obtained from a single matrix diagonalization, and so one of the cost advantages of wave functions over Green functions is not available in the present situation.

In the usual way, the Green function $G$ for the present problem is that which solves the inhomogeneous Schrödinger equation corresponding to Eq. (5). Expanding $G\left(\mathbf{r}, \mathbf{r}^{\prime} ; E\right)$ in the basis set $\left\{\chi_{\mu}(\mathbf{r})\right\}$,

$$
G\left(\mathbf{r}, \mathbf{r}^{\prime} ; E\right)=\sum_{\mu \mu^{\prime}} g(E)_{\mu \mu^{\prime}} \chi_{\mu}(\mathbf{r}) \chi_{\mu^{\prime}}^{*}\left(\mathbf{r}^{\prime}\right),
$$

the Green-function expansion coefficients may be shown to satisfy the matrix equation,

$$
\sum_{\mu^{\prime \prime}}\left[H_{\mu \mu^{\prime \prime}}+G_{0}^{-1}(E)_{\mu \mu^{\prime \prime}}-E O_{\mu \mu^{\prime \prime}}\right] g(E)_{\mu^{\prime \prime} \mu^{\prime}}=\delta_{\mu \mu^{\prime}},
$$

where contributions to the matrix elements from the Hamiltonian, embedding potential, and overlap terms are 


$$
\begin{gathered}
H_{\mu \mu^{\prime}}=\int_{I} \mathbf{d}^{3} \mathbf{r} \chi_{\mu}^{*} H \chi_{\mu^{\prime}}+\frac{1}{2} \int_{S} \mathbf{d}^{2} \mathbf{r}_{S} \chi_{\mu}^{*} \frac{\partial}{\partial n_{S}} \chi_{\mu^{\prime}}, \\
G_{0}^{-1}(E)_{\mu \mu^{\prime}}=\int_{S} \mathbf{d}^{2} \mathbf{r}_{S} \int_{S} \mathbf{d}^{2} \mathbf{r}_{S}^{\prime} \chi_{\mu}^{*} G_{0}^{-1}(E) \chi_{\mu^{\prime}}, \\
O_{\mu \mu^{\prime}}=\int_{I} \mathbf{d}^{3} \mathbf{r} \chi_{\mu}^{*} \chi_{\mu^{\prime}} .
\end{gathered}
$$

We emphasize that the theory developed so far does not introduce any additional approximation beyond the singleparticle model. In the usual manner, Eq. (7) is solved selfconsistently following density functional theory ${ }^{8,9}$ in the local density approximation, ${ }^{10}$ as used here, or if desired by using gradient-corrected extensions to the LDA. ${ }^{11}$ In practice, further approximations occur in the solution, due to truncation of the basis set at a finite basis set size - introducing an error which can be monitored and, in principle, systematically reduced to any arbitrary level of precision required - and through the choice of embedding volume within which the solution is obtained self-consistently. In principle, this too can be systematically increased and the error reduced to any desirable level.

\section{The embedded region}

We now consider the actual geometry of the embedded region suitable for the adsorption problem. In previous applications (e.g., Refs. 25,26,28-30) of embedding methods to the study of surface properties, the embedded region has consisted of the selvedge region of the crystal surface (the top layer or two) and a vacuum portion. In these studies, the focus of attention has been on properties of the clean surface, which is characterized by the translational invariance symmetry parallel to the surface and for which the problem is simplified through the use of two-dimensional Bloch wave functions. Here, the presence of an adsorbate on the surface destroys this invariance to translation across the surface, but represents a perturbation to the crystal, which involves a relatively small localized volume in the vicinity of the adsorbate. This suggests that for the embedding region, one should use a limited volume around the adsorbate, but one large enough to contain that part of the substrate that is perturbed by the impurity. The reference system used to calculate the embedding potential is the clean surface.

A second factor influencing the choice of embedding region is the question of the expansion of the embedding potential and the subsequent determination of the electronic structure of the adsorbate on the surface. Both are considerably simplified if the embedding potential can be expanded in a complete, orthogonal set of surface basis functions on $S$, leading to an efficient and compact representation. This also simplifies the determination of the Green-function matrix elements. These two requirements are certainly satisfied through the choice of a spherical embedded region, where the spherical harmonic basis set may be used for surface expansions.

\section{The embedding potential}

The embedding potential may be determined from either the wave function [for example, by inverting Eq. (3)] or the Green function $^{31}$ of the unperturbed system, which in the present case is the clean surface. The clean surface is a system which may itself be usefully studied by embedding, ${ }^{25,26,29}$ and so for the current discussion, we assume a knowledge of the Green function rather than the wave function.

We denote this Green function $G_{M}\left(\mathbf{r}, \mathbf{r}^{\prime} ; E\right)$. This is normally available in an expansion, using a basis appropriate to the clean surface geometry, such as plane or evanescent waves normal to the surface and Bloch waves along the surface plane. ${ }^{25}$ As described in Sec. II A, the embedding potential is the surface inverse on $S$ of the clean substrate Green function, which has zero normal derivative on $S$, the embedding surface. This may be derived from $G_{M}$, which will, in general, satisfy some other boundary conditions (usually periodic boundary conditions parallel to the substrate and causal boundary conditions normal to the surface, or, if $G_{M}$ is obtained from a supercell calculation, periodic boundary conditions both parallel and normal to the substrate surface) by matching Green-function methods. ${ }^{32}$ By suitable transformation, $G_{M}$ may be expanded on the embedding surface $S$, the surface of the embedded sphere, which has radius $a$, in spherical harmonics (energy dependence is now implicit):

$$
G_{M}\left(\mathbf{r}_{S}, \mathbf{r}_{S}^{\prime}\right)=\sum_{L L^{\prime}} \Gamma_{L L^{\prime}} Y_{L}(\Omega) Y_{L^{\prime}}^{*}\left(\Omega^{\prime}\right)
$$

where $L$ is the composite angular momentum index $L=(\ell, m)$, and $\Omega$ the solid angle $\Omega \equiv(\theta, \phi)$. Similarly, the normal derivative of $G_{M}$ may be expanded on the surface, with coefficients $\left\{\Gamma_{L L^{\prime}}^{\prime}\right\}$, and the matching Green-function method then shows that the coefficients $\left\{\mathscr{G}_{0 L L^{\prime}}^{-1}\right\}$ of the surface expansion of the embedding potential,

$$
G_{0}^{-1}\left(\mathbf{r}_{S}, \mathbf{r}_{S}^{\prime}\right)=\sum_{L L^{\prime}} \mathscr{G}_{0 L L^{\prime}}^{-1} Y_{L}(\Omega) Y_{L^{\prime}}^{*}\left(\Omega^{\prime}\right),
$$

may be found from the matrix equation,

$$
\mathscr{G}_{0}^{-1}=\Gamma^{-1}\left[1+\frac{a^{2}}{2} \Gamma^{\prime}\right] .
$$

The evaluation of the matrix elements $\Gamma$ and $\Gamma^{\prime}$ from $G_{M}$ involves integration over the Brillouin zone and summations over reciprocal-lattice vectors, and along with the Greenfunction expansion is discussed in some detail in Appendix A.

We note that there is no problem in obtaining the embedding potential for a substrate described by nonlocal pseudopotentials, even if the embedding surface should cut through the core of a pseudopotential. This is because the pseudowave-function is still a local quantity, and it is this which determines the embedding potential, either directly or through the Green function. Naturally, the closer the embedding surface approaches an atomic core, the more structure there will be in the substrate wave functions, and so the larger the number of basis functions that will be required in 
the expansions of $G_{M}$ and $G_{0}^{-1}$. This will also be true should the embedding potential be determined from an all-electron description of the substrate (e.g., based upon the muffin-tin construct), which will most probably require an augmented basis set description for the surface expansions.

The embedding potential contains all the information regarding the substrate which will enter into the solution of the Schrödinger equation for the perturbed region. It does not depend upon the contents of the embedded volume, and needs to be evaluated only once for a given substrate and choice of embedding volume. Hence it is worthwhile to evaluate it to high accuracy. Once this has been done an arbitrary perturbation may be introduced into the embedded region, and the electronic structure obtained from a calculation for this region with the embedding potential acting as a boundary condition. The solution so obtained will be entirely equivalent to having solved the problem of the combined substrate+adsorbate system assuming complete basis set convergence and that the perturbation in the charge density and potential are restricted to the embedded volume.

\section{E. Basis set and matrix elements}

This embedding approach is flexible enough to allow for an arbitrary choice of the basis set, but to proceed, it is necessary to make a concrete choice. For the present study, in which a single adsorbate atom treated in the pseudopotential approximation is considered on jellium, a convenient basis is constructed from spherical Bessel functions for radial variation and spherical harmonics for angular variation:

$$
\chi_{\mu}(\mathbf{r})=\chi_{n, \ell, m}(r, \Omega)=j \curlywedge\left(k_{n} r\right) Y_{L}(\Omega) .
$$

The argument of the Bessel function is chosen to be $k_{n}=n \pi / \tilde{a}$, where $\tilde{a}>a, a$ being the radius of the embedding volume. This gives a range of values of amplitude and derivative on the surface of the sphere and so does not prejudice or constrain the description of the boundary condition. The composite index $\mu$ represents both radial $-n-$ and angular - $\ell, m-$ indices. The basis set consists of the $n_{M} \times\left(\ell_{M}+1\right)^{2}$ functions with $1 \leqslant n \leqslant n_{M}$ and $0 \leqslant \ell \leqslant \ell_{M}$. Within the spherical geometry, this basis set may be viewed as the natural relative of the familiar plane wave basis set used in more conventional electronic structure calculations, and indeed it retains many of the benefits of the latter (a competitive alternative would use Gaussians for the radial variation). Note that a similar basis will also be suitable for studies which described the substrate by pseudopotentials, and also for molecular adsorbates. More localized basis functions will be needed for all-electron calculations, for which a suitable generalization of the linear augmented plane waves basis $^{25}$ is an obvious choice.

The matrix elements in Eq. (7) can now be evaluated. The overlap matrix is

$$
O_{\mu \mu^{\prime}}=\delta_{L L^{\prime}} \int_{0}^{a} r^{2} j \ell\left(k_{n} r\right) j_{\ell^{\prime}}\left(k_{n^{\prime}} r\right) d r .
$$

The Hamiltonian matrix may be written as three contributions, $H=T+D+V$. The first, the kinetic energy, is straightforward, as the basis functions are eigenstates of the kinetic energy operator with eigenvalue $k_{n}^{2} / 2$ :

$$
T_{\mu \mu^{\prime}}=\frac{k_{n^{\prime}}^{2}}{2} O_{\mu \mu^{\prime}}
$$

The second contribution to the Hamiltonian is that due to the normal derivative, the final term in the first line of Eq. (8). This term is

$$
D_{\mu \mu^{\prime}}=\delta_{L L^{\prime}} \frac{a}{2} j \ell\left(k_{n} a\right)\left[\ell j \ell\left(k_{n^{\prime}} a\right)-k_{n^{\prime}} a j \ell+1\left(k_{n^{\prime}} a\right)\right],
$$

making use of recurrence relations satisfied by the Bessel functions. ${ }^{33}$

We now consider the final contribution to the Hamiltonian, $V$, due to the potential. We assume that the potential is a combination of local and nonlocal terms. The former includes the exchange-correlation potential, the electrostatic potential, and the local part of the ionic potential. The latter is the nonlocal component of the pseudopotential. In allelectron calculations this latter contribution would be zero.

The sum of all the local terms may be expanded within the embedded sphere as

$$
V(\mathbf{r})=\sum_{L^{\prime \prime}} V_{L^{\prime \prime}}(r) Y_{L^{\prime \prime}}(\Omega)
$$

The radial components $V_{L}(r)$ are tabulated on a grid and include contributions from the ionic core, known analytically, the Coulomb contribution, which is found from the solution of the Poisson equation (this reduces to the solution of a radial problem for each $L$ component), and the exchange correlation potential, which is numerically evaluated by fitting its angular variation via a special directions expansion.

The contribution to the Hamiltonian matrix elements from the local potential is

$$
V_{\mu \mu^{\prime}}^{\mathrm{LOC}}=\sum_{L^{\prime \prime}} S_{L^{\prime} L^{\prime \prime}}^{L} \int_{0}^{a} r^{2} j\left(k_{n} r\right) V_{L^{\prime \prime}}(r) j_{\ell^{\prime}}\left(k_{n^{\prime}} r\right) d r
$$

where $S_{L^{\prime} L^{\prime \prime}}^{L}$ is the integral of three spherical harmonics,

$$
S_{L^{\prime} L^{\prime \prime}}^{L}=\int_{4 \pi} d \Omega Y_{L}^{*}(\Omega) Y_{L^{\prime}}(\Omega) Y_{L^{\prime \prime}}(\Omega)
$$

Only values of $L, L^{\prime}$, and $L^{\prime \prime}$ satisfying certain conditions give nonzero $S_{L^{\prime} L^{\prime \prime}}^{L} \cdot{ }^{34}$ One consequence is that only components of the potential $V_{L^{\prime \prime}}$ for which $\ell^{\prime \prime} \leqslant 2 \ell_{M}$ need be retained.

The contribution from the nonlocal terms is more troublesome, as these have a natural origin, which is the position of the adsorbate, $\mathbf{R}_{a}$, rather than the center of the embedded region, which is the origin of the basis functions. We have used $a b$ initio pseudopotentials of the BacheletHamann-Schlüter ${ }^{35}$ type, which have the form (neglecting the spin-orbit interaction)

$$
\hat{V}_{\mathrm{ps}}^{\mathrm{ion}}\left(r_{a}, \Omega_{a}, \Omega_{a}^{\prime}\right)=\sum V_{\ell}^{\mathrm{ion}}\left(r_{a}\right) \sum_{m=-\ell}^{\ell} Y_{L}\left(\Omega_{a}\right) Y_{L}^{*}\left(\Omega_{a}^{\prime}\right),
$$


where $r_{a}=\left|\mathbf{r}-\mathbf{R}_{a}\right|$ and $\Omega_{a}$ is the solid angle subtended by $\mathbf{r}$ relative to the origin $\mathbf{R}_{a}$. The radial components may be decomposed as

$$
V_{\ell}^{\mathrm{ion}}(r)=V_{\text {core }}(r)+\Delta V_{\ell}^{\mathrm{ion}}(r)
$$

where the first term, the long-range local Coulomb contribution, may be reexpanded about the center of the embedded sphere and then combined with the other local contributions above. The contributions to the Hamiltonian matrix elements arising from the short-ranged $\ell$-dependent terms are found by reexpanding the basis functions about the adsorbate position, using

$$
j \ell\left(k_{n} r\right) Y_{L}(\Omega)=\sum_{L^{\prime}} D_{L^{\prime}}^{L}\left(k_{n}, \mathbf{R}_{a}\right) j_{\ell^{\prime}}\left(k_{n} r_{a}\right) Y_{L^{\prime}}\left(\Omega_{a}\right),
$$

where

$$
D_{L^{\prime}}^{L}(k, \mathbf{R})=4 \pi \sum_{L^{\prime \prime}} i^{\left(\ell^{\prime}-\ell-\ell^{\prime \prime}\right)} j_{\ell^{\prime \prime}}(k R) S_{L L^{\prime \prime}}^{L^{\prime}} Y_{L^{\prime \prime}}^{*}\left(\Omega_{\mathbf{R}}\right),
$$

to give

$$
\begin{aligned}
V_{\mu \mu^{\prime}}^{\mathrm{NL}}= & \sum_{L^{\prime \prime}} D_{L^{\prime \prime}}^{L *}\left(k_{n}, \mathbf{R}_{a}\right) D_{L^{\prime \prime}}^{L^{\prime}}\left(k_{n^{\prime}}, \mathbf{R}_{a}\right) \\
& \times \int_{0}^{+\infty} r^{2} j_{\ell^{\prime \prime}}\left(k_{n} r\right) \Delta V_{\ell^{\prime \prime}}^{\mathrm{ion}}(r) j_{\ell^{\prime \prime}}\left(k_{n^{\prime}} r\right) d r .
\end{aligned}
$$

Note that, in practice, the short range of the radial components of the nonlocal potential introduces a finite cutoff to this radial integral.

Finally, the matrix elements of the embedding potential are obtained in a straightforward way using the expansion (10) and exploiting the orthogonality of the spherical harmonics:

$$
G_{0}^{-1}(E)_{\mu \mu^{\prime}}=a^{4} \mathscr{G}_{0}^{-1}(E)_{L L^{\prime}} j\left(k_{n} a\right) j_{\ell^{\prime}}\left(k_{n^{\prime}} a\right) .
$$

\section{QUANTITIES OF PHYSICAL INTEREST}

Both in the self-consistent solution of the Schrödinger equation (5) and as a quantity of physical interest, the electron charge density $\rho_{\mathrm{el}}(\mathbf{r})$ plays a major role in the theory. We determine the charge density from the local density of states (LDOS), $\sigma(\mathbf{r}, E)$, which is related to the Green function by

$$
\sigma(\mathbf{r}, E)=\frac{1}{\pi} \operatorname{Im} G(\mathbf{r}, \mathbf{r} ; E+i \epsilon),
$$

where $i \epsilon$ is a small imaginary energy. The charge density is found by integrating over the occupied states. This is most economically performed by contour integration, exploiting the analyticity of the Green function in the upper half plane:

$$
\rho_{\mathrm{el}}(\mathbf{r})=\frac{1}{\pi} \operatorname{Im} \int_{c} d z G(\mathbf{r}, \mathbf{r} ; z)
$$

with the curve $c$ beginning below the lowest occupied state ${ }^{36}$ and returning to the real energy axis at the Fermi energy $E_{F}$. The density of states (DOS) is found from the spatial integral of the LDOS,

$$
\sigma(E)=\int \mathbf{d}^{3} \mathbf{r} \sigma(\mathbf{r}, E)
$$

a result which clearly depends upon the integration volume. Using the generalized phase shift (GPS), ${ }^{37}$ it is possible to determine the change in the DOS in the whole space, due to the presence of the adsorbate. This includes changes within the embedded region and the substrate. The change in the total number of electrons with energies less than $E$ is given by

$$
\Delta N(E)=-\frac{1}{\pi} \operatorname{Im} \ln \operatorname{det}\left(\frac{H+G_{0}^{-1}-E O}{H_{c}+G_{0}^{-1}-E O}\right) \equiv \int d E \Delta \sigma(E),
$$

where $H_{c}$ is the Hamiltonian of the clean surface. $\Delta N(E)$ can be numerically differentiated to yield $\Delta \sigma(E)$, the change in the density of states of the whole system, which is a quantity independent of the radius of the embedding sphere used in the calculation, once it is sufficiently large.

Finally, we are interested in the total energy of the system, and, in particular, the interaction energy $\mathscr{E}\left(\mathbf{R}_{a}\right)$ of the atom with the surface, which is represented by the difference between the total energy of the interacting system and the reference energy of the clean surface and the isolated atom (for more details see Appendix B). This function of adsorbate position represents the adiabatic potential energy curve for the adsorption process. Using a grand-canonical energy functional, ${ }^{38-40}$ we find $\mathscr{E}\left(\mathbf{R}_{a}\right)$ to be

$$
\begin{gathered}
\mathscr{E}\left(\mathbf{R}_{a}\right)=\langle\Delta T\rangle+\langle\Delta U\rangle-E_{F} \Delta Q \\
\langle\Delta T\rangle=\int_{-\infty}^{E_{F}} d E E \Delta \sigma(E)-\int_{I} \mathbf{d}^{3} \mathbf{r} \rho_{\mathrm{el}}(\mathbf{r}) V_{\mathrm{eff}}(\mathbf{r}), \\
\langle\Delta U\rangle=\frac{1}{2} \int_{I} \mathbf{d}^{3} \mathbf{r} \int_{R^{3}} \mathbf{d}^{3} \mathbf{r}^{\prime} \frac{\rho(\mathbf{r}) \rho\left(\mathbf{r}^{\prime}\right)}{\left|\mathbf{r}-\mathbf{r}^{\prime}\right|} \\
+\int_{I} \mathbf{d}^{3} \mathbf{r} \rho_{\mathrm{el}}(\mathbf{r}) \varepsilon_{\mathrm{xc}}\left(\rho_{\mathrm{el}}(\mathbf{r})\right)
\end{gathered}
$$

where $V_{\text {eff }}(\mathbf{r})$ is the effective potential felt by the electrons, with electrostatic and exchange-correlation contributions in addition to the pseudopotential. $\rho(\mathbf{r})$ is the total charge density, and $\Delta Q$ denotes the excess $(\Delta Q>0)$ or the deficit $(\Delta Q<0)$ of electronic charge in the whole system $(I+I I)$ calculated by the GPS method. Although small for sufficiently large embedding volumes, this provides a significant contribution to the total energy, which is accounted for within the grand-canonical functional by the additional energy contribution $E_{F} \Delta Q$, in effect subtracting/adding the excess/missing charge at the Fermi energy of the substrate, which acts as a reservoir of electrons to flow into and out of the whole system. ${ }^{41}$ 


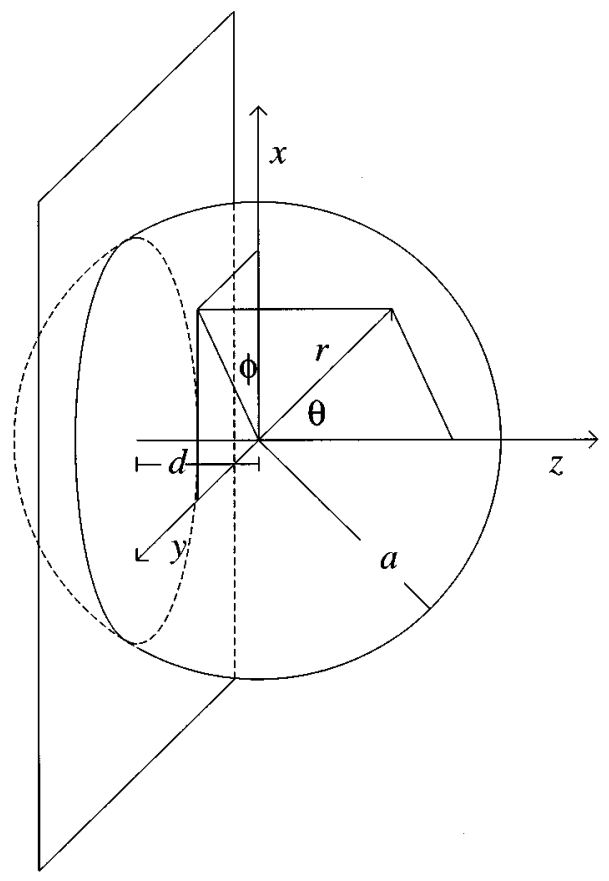

FIG. 2. The embedded sphere and coordinate system used in our method. The semi-infinite background fills the left portion of the space. For details see the text.

\section{APPLICATIONS}

As the first application of this embedding method, we consider a single isolated atom on a jellium surface, this problem representing a suitable nontrivial system for testing - a critical comparison can be made with the well known (and often revisited) results of Lang and Williams. ${ }^{7}$ We initially require a self-consistent solution for the adsorbate-free surface from which the embedding potential may be determined. For this we perform a separate calculation embedding a selvedge region onto a semi-infinite jellium substrate, and the embedding potential is constructed by using Eqs. (10) and (11) on a sphere of radius $a$, centered a distance $d$ above the jellium edge. The geometry is indicated in Fig. 2.

To test the quality of the embedding potential, we first solved Eq. (7) self-consistently within the embedding region with no adatom. This "empty lattice" test also provides an assessment of the implementation of the theory, and we found that the calculated behavior of physical quantities (charge density, DOS, effective one-electron potential, etc.) for several values of the Wigner radius $r_{s}$ were in very good agreement with those found from one-dimensional calculations of the same systems, and that they merged nicely into those of bulk jellium.

We have next considered a $\mathrm{Si}$ atom chemisorbed on $\mathrm{Al}$, modeled by jellium with $r_{s}=2.07 a_{0} \cdot{ }^{42}$ A number of test calculations indicated that a sphere of radius $a=7 a_{0}$ at a distance $d=1 a_{0}$ from the jellium edge was sufficiently large. For all the parameters of the calculation see Table I. Detailed results for this $\mathrm{Si}-\mathrm{Al}$ system have been given by Lang and Williams in Ref. 7, using a method in which the adsorbate system wave functions are matched onto those of bulk jellium; our embedding method may be used for any substrate.
TABLE I. Parameters used in the calculations. $a$ is the embedding sphere radius, $E^{\text {cut }}$ is the kinetic energy cutoff, and $\left(\ell_{M}+1\right)^{2}$ and $n_{M}$ are the number of spherical and radial basis functions, respectively. The values given have been arrived at on the basis of extensive convergence tests, e.g., increasing $n_{m}$ to 18 changes the $\mathrm{N}$ adsorption energy by $<0.01 \mathrm{eV}$, or decreasing $\ell_{m}$ by 1 makes a negligible change to the DOS or induced DOS as plotted.

\begin{tabular}{lcccc}
\hline \hline Physical system & $a / a_{0}$ & $E^{\text {cut }} /$ Ry & $\ell_{M}$ & $n_{M}$ \\
\hline Clean Al surf. & 7 & 23 & 8 & 12 \\
Si on Al & 7 & 30 & 8 & 14 \\
N on Al & 7 & 44 & 8 & 16 \\
\hline
\end{tabular}

The other main differences between the two approaches are (i) we use a pseudopotential ${ }^{35}$ for describing the atom, instead of an all-electron potential used in Ref. 7. (ii) Lang and Williams ${ }^{7}$ consider some effects caused by the long-range charge disturbance, due to the adatom by including a correction in the self-consistent potential at each iteration, while our calculation is performed purely within the embedded region. (iii) We use the more accurate exchange-correlation potential from Ref. 43. (iv) Finally, we compute the adsorption energy by the grand-canonical functional.

Figures 3 and 4 show the calculated charge density and density difference arising from the adsorption of $\mathrm{Si}$ on $\mathrm{Al}$ at distance $d_{\mathrm{eq}}=2.3 a_{0}$ from the jellium edge (the equilibrium position found in Ref. 7). In the former, the radius of the embedding sphere, beyond which the calculated charge density has no physical relevance, is clearly visible, but for the difference plot this is not the case, due to the localization of the charge perturbation to within the embedding volume. Both plots agree well with those in Ref. 7, and the minor differences near the nucleus position can be attributed to the different treatments of the ion core.

We show the calculated adsorption-induced DOS in Fig. 5 for several atom-surface distances. This quantity represents the difference between the density of states of the surface + adsorbate system and that of the clean surface alone, hence revealing how the distribution of electronic states has changed. On the scale of Fig. 5, both the induced DOS calculated within the embedded volume (region $I$ ), which is plotted, and that found from the GPS for the total space

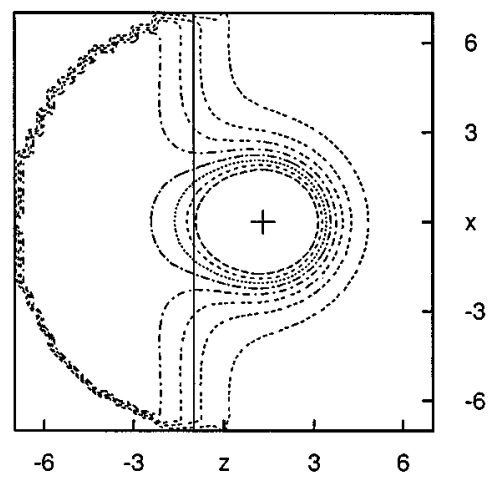

FIG. 3. Contour plot of the electron charge $\rho_{\mathrm{el}}(\mathbf{r})$ for $\mathrm{Si}$ on an Al-jellium surface at the distance $d_{\mathrm{eq}}=2.3 a_{0}$. In the outermost contour $\rho_{\mathrm{el}}=0.004$ a.u.; successive contours increase by 0.006 a.u. $x, z$ is in units of $a_{0}$. 


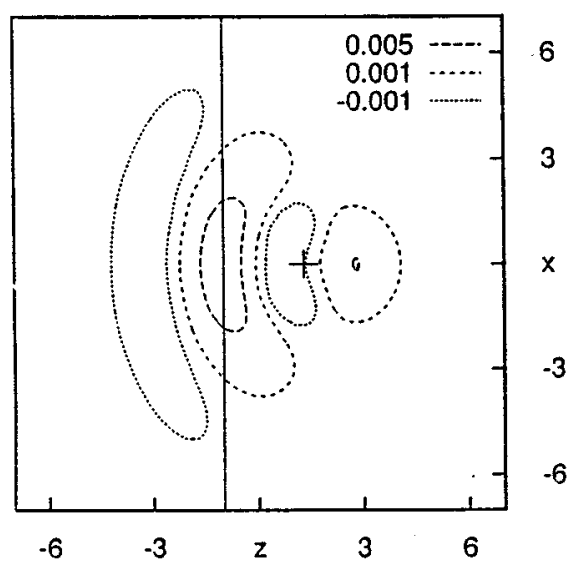

FIG. 4. Contour plot of the displacements of the electron charge for $\mathrm{Si}$ on an Al-jellium surface at $d_{\mathrm{eq}}=2.3 a_{0} . x, y$ is in units of $a_{0}$.

$(I+I I)$ are essentially indistiguishable. The inset, showing results for $d_{\mathrm{eq}}=2.3 a_{0}$, compares very well with the previous calculation reported in Ref. 7. The two resonances, which are more pronounced and shift to higher energies when the atom gets further from the surface, correspond to the $3 s$ and $3 p$ atomic levels. Figure 6 shows the positions of the $3 p$ peak of the $m=0(\sigma$-like $)$ and $|m|=1(\pi$-like $)$ components of the DOS at various distances from the surface. The splitting, in agreement with the results of Ref. 7, arises from the breaking of the spherical symmetry of the atom by the surface.

We now consider the interaction energy of the $\mathrm{Si}-\mathrm{Al}$ system [Eqs. (B3)-(B7)]. For consistency, we determine the atomic energy $E^{\text {at }}$ (at infinite distance from the surface) by embedding the atom in vacuum. We have checked that an embedding sphere of radius $a=12 a_{0}$, gives electronic properties of the atom, which coincide with those obtained by solving the standard radial Kohn-Sham problem, while for $a=7 a_{0}$, we find they only differ negligibly. Therefore, we compute $E^{\text {at }}$ within a sphere of the same radius as that of the

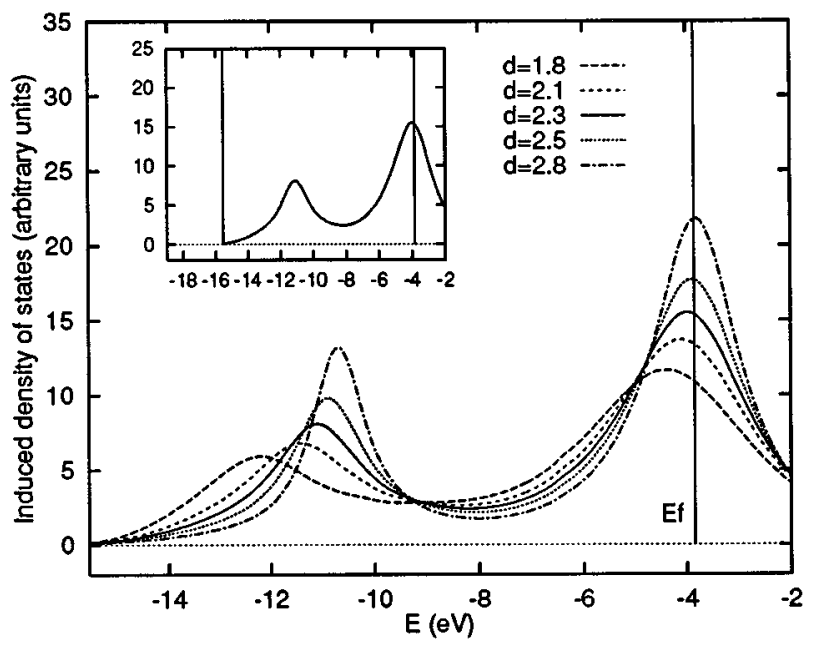

FIG. 5. Induced DOS of $\mathrm{Si}$ on an Al-jellium surface at several atom-surface distances. The vacuum zero energy is the reference energy level. In the inset, the induced DOS at the distance $d_{\text {eq }}=2.3 a_{0}$ is shown.

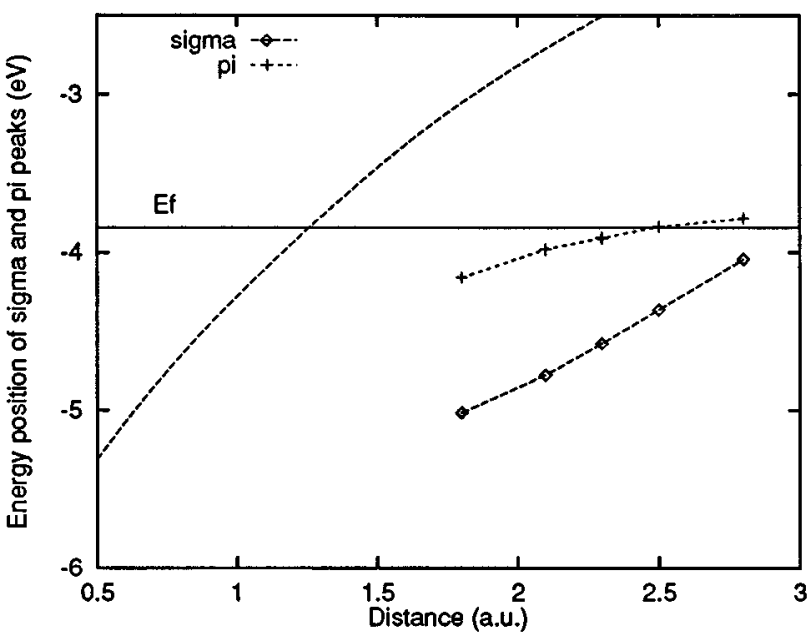

FIG. 6. The $m=0$ ( $\sigma$-like) and $|m|=1$ ( $\pi$-like) contributions to the induced DOS of $\mathrm{Si}$ on an Al-jellium surface (see previous figure). The dashed line is the effective one-electron potential of the clean metal.

atom-surface embedded region, and we also position the atom within the sphere at the same position as for the surface calculation. By this procedure, we minimize errors arising from the choice of a smaller radius.

We note that $a b$ initio total energy calculations are usually a difficult task for any impurity problem in an external metal host, due to the long-range character of the Friedel oscillations, induced in our case by the adatom. As a consequence, the perturbation cannot be fully localized to the embedded region. Perfect screening will only occur if an infinitely extended embedded region is used, and so some violation of charge neutrality in the whole system is expected. The excess/deficit of electron charge is measured (Sec. III) by $\Delta Q=\left[\Delta N\left(E_{F}\right)-Z\right], Z$ being the number of the atom valence electrons. For $\mathrm{Si}$ on $\mathrm{Al}$ at the adatom-metal equilibrium distance from the jellium edge $d_{\mathrm{eq}}=2.3 a_{0}$, we find $\Delta N\left(E_{F}\right)=4.072$ and so $\Delta Q=0.072$, while the local excess

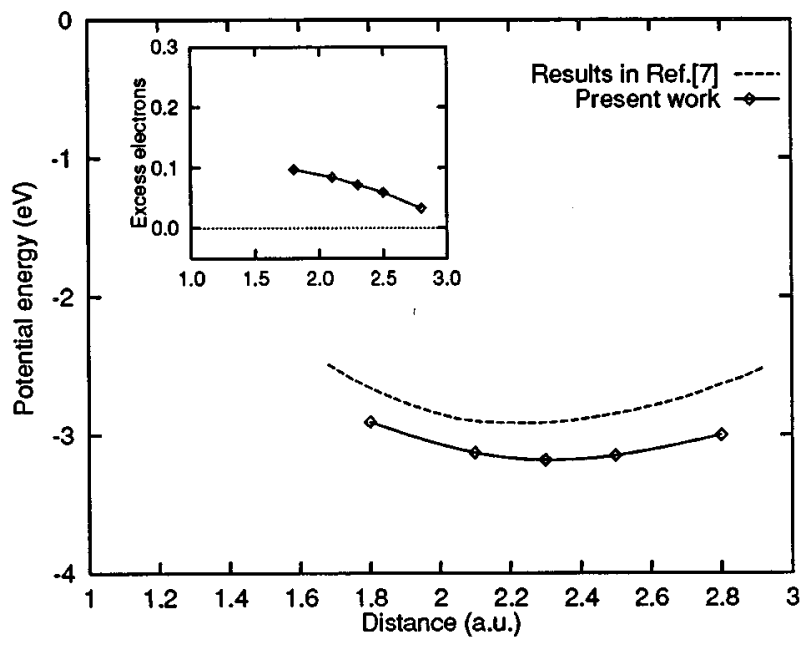

FIG. 7. Adsorption energies of $\mathrm{Si}$ on $\mathrm{Al}$, as functions of the atom-surface distance. In the inset $\Delta Q$, as a function of the atomsurface distance, is shown. 


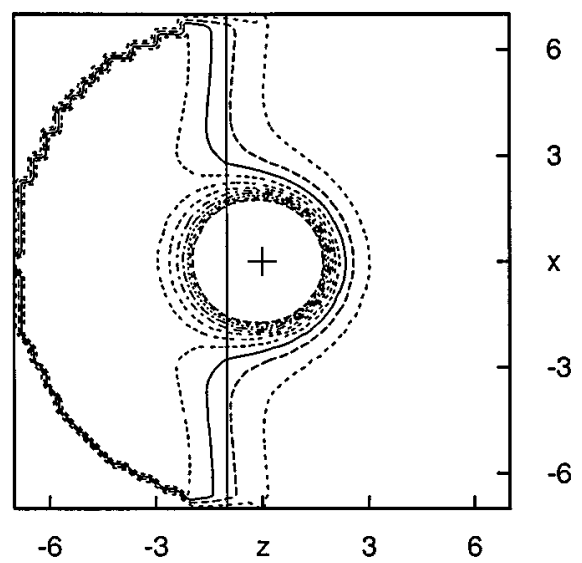

FIG. 8. Contour plot of the electron charge $\rho_{\mathrm{el}}(\mathbf{r})$ for $\mathrm{N}$ on an Al-jellium surface at the equilibrium distance $d_{\mathrm{eq}}=1.0 a_{0}$. In the outermost contour $\rho_{\mathrm{el}}=0.004$ a.u.; successive contours increase by 0.006 a.u. $x, y$ in units of $a_{0}$.

of the electron charge in the embedded region is found to be $\Delta Q_{I}=0.09$ (with the calculation parameters reported in Table I). The excess of electron charge in the whole system $\Delta Q$, as function of the atom-surface distance, is shown in the inset of Fig. 7. This effect is taken into account in the adsorption energies by the grand-canonical formalism (see Sec. III and Ref. 40). The resulting adsorption energies of a $\mathrm{Si}$ adatom at various distances from the jellium edge are shown in Fig. 7, with those of Lang and Williams. ${ }^{7}$ We observe good agreement between the Si-jellium energies calculated in this paper and those in Ref. 7, even though the selfconsistent Schrödinger equation is solved within two very different frameworks. This agreement is to be expected given the agreement between the charge contours and DOS obtained with the two methods.

We now consider $\mathrm{N}$ on the same substrate, $\mathrm{Al}$ described by jellium. This is one of the very few first row elements, the electronic adsorption properties on simple metals of which have yet to be the subject of first-principles calculations. Furthermore, it has been shown experimentally that the interac-

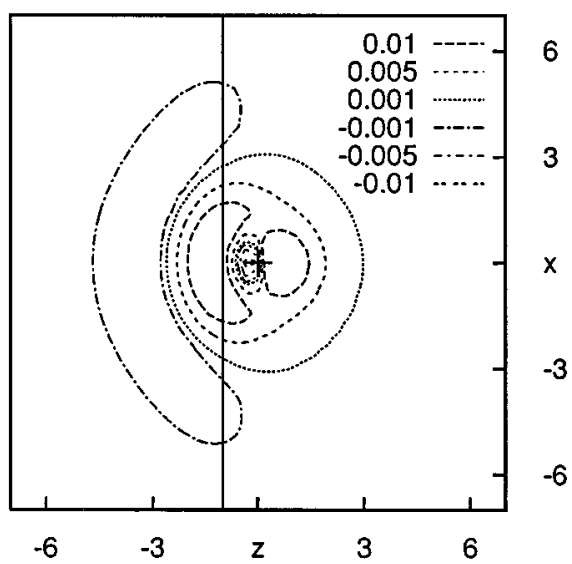

FIG. 9. Contour plot of the displacements of the electron charge for $\mathrm{N}$ adsorbed on an Al-jellium surface at $d_{\mathrm{eq}}=1.0 a_{0} . x, y$ in units of $a_{0}$.

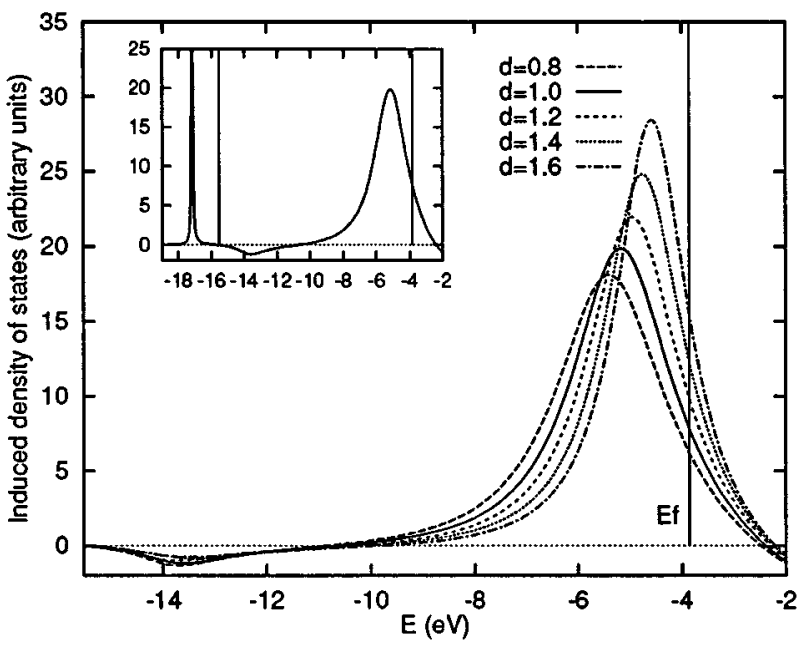

FIG. 10. Induced DOS of $\mathrm{N}$ on an Al-jellium surface at several atom-surface distances. The vacuum zero energy is the reference energy level. In the inset, the induced DOS at the equilibrium distance $d_{\mathrm{eq}}=1.0 a_{0}$ is shown. The narrow peak on the left represents the $2 s$ level.

tion of $\mathrm{N}_{2}^{+}$ions with an $\mathrm{Al}$ surface leads to formation of an AlN film, hence with $\mathrm{N}$ atomic adsorption. ${ }^{44,45}$ These points motivate our theoretical investigation of $\mathrm{N}$ adsorption on jellium, as well as the quest for a harder test of our method than treating a $\mathrm{Si}$ adatom.

As the $\mathrm{N}$ atom pseudopotential is considerably deeper and more compact than that of $\mathrm{Si}$, a greater numerical effort is required, and, in particular, more radial basis functions are needed, as shown in Table I. The contour plots of the charge density and of the displacements of the electron charge at the calculated atom-surface bond equilibrium distance of $d_{\mathrm{eq}}=1.0 a_{0}$ from the jellium edge (see below), are shown in Figs. 8 and 9, respectively. Owing to the larger electronegativity of $\mathrm{N}$ compared to $\mathrm{Si}$, these plots more closely resemble those previously computed for a $\mathrm{Cl}$ adatom on jellium. ${ }^{7}$ For

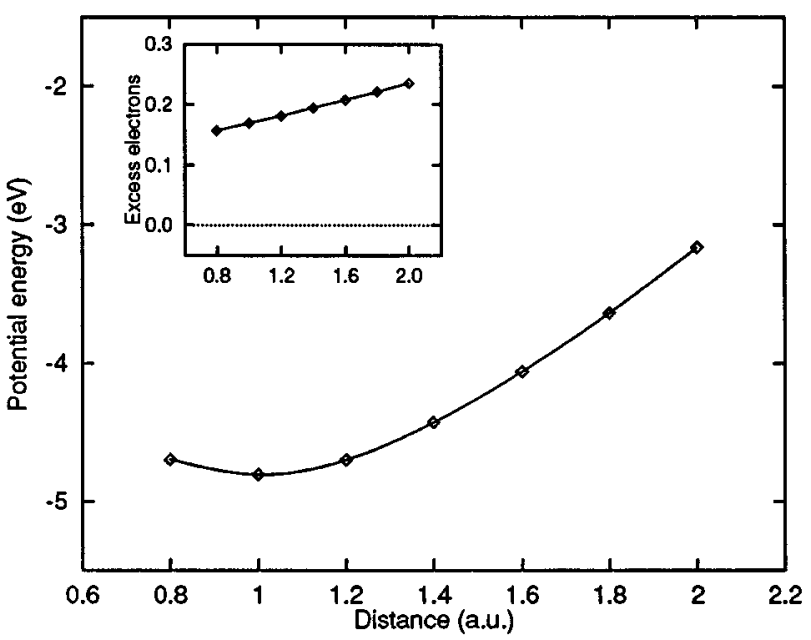

FIG. 11. Adsorption energies of $\mathrm{N}$ on $\mathrm{Al}$, as functions of the atom-surface distance. In the inset $\Delta Q$, as a function of the atomsurface distance, is shown. 
both $\mathrm{Cl}$ and $\mathrm{N}$, there is an enhancement of the electronic charge in the atomic region, indicating that the $\mathrm{N}$-jellium (Al-like) adsorption bond has a more ionic character than the Si-jellium bond. The induced DOS in Fig. 10 calculated at various distances from the jellium edge shows a pronounced peak, due to the $2 p$ resonance level of $\mathrm{N}$. The behavior of the resonance as a function of distance is the same as that of the $3 p$ resonance of $\mathrm{Si}$, but in the case of $\mathrm{N}$, lies further below the Fermi energy, another indication of the predominantly ionic character of the $\mathrm{N}$-jellium bond. At lower energies, near $-14 \mathrm{eV}$, the induced DOS becomes slightly negative, indicating a redistribution of states to higher energies upon $\mathrm{N}$ adsorption. A similar effect occurs with different adsorbates. ${ }^{7}$ However, as well as lying deep in energy, this feature exhibits only minor changes with varying adsorbate height and so does not influence the adsorption process significantly. The inset of Fig. 10 shows the DOS at the $\mathrm{N}$-jellium equilibrium distance $d_{\mathrm{eq}}=1.0 a_{0}$. At $-17 \mathrm{eV}$, below the substrate continuum, the sharp N $2 s$ level may also be seen. With varying $d$, this level trivially follows the effective potential of the clean surface. Again, lying so deep in energy, this state does not influence the adsorption process.

Owing to the more reactive nature of $\mathrm{N}$, the perturbation induced by the adsorbate is less well screened than that of $\mathrm{Si}$, and this is evident in the lack of perfect charge neutrality in our calculation. At the $\mathrm{N}$-surface equilibrium position, we calculate $\Delta N\left(E_{F}\right)=5.169, \Delta Q=0.169$, and $\Delta Q_{I}=0.216$ for an embedding sphere of radius $a=7 a_{0}$. Its variation with $d$ is shown in the inset to Fig. 11. With $a=7.5 a_{0}$, we obtain a decrease of $\Delta Q$ and $\Delta Q_{I}$ a little larger than $10 \%$. The adsorption energy for $\mathrm{N}$ on jellium, computed by the grandcanonical functional to correct (to first order) for the nonzero value of $\Delta Q$, is shown in Fig. 11 as a function of atomsurface distance. At $d_{\mathrm{eq}}=1.0 a_{0}$, the $\mathrm{N}$-Al adsorption energy is $E_{\mathrm{ads}} \sim-4.8 \mathrm{eV}$, corresponding to a more strongly bonded system than $\mathrm{Si}-\mathrm{Al}$. This value of $E_{\text {ads }}$ is comparable with that found by Lang and Williams for $\mathrm{O}$ on jellium, $-5.4 \mathrm{eV}{ }^{7}$ The smaller value of $d_{\text {eq }}$ compared with $\mathrm{Si}$ is also comparable with the distance found by Lang and Williams for $\mathrm{O}$ on jellium $\left(1.1 a_{0}\right){ }^{7}$ and is consistent with the small size of the $\mathrm{N}$ atom in chemisorption-it tends to tuck into the surface. ${ }^{46}$

\section{CONCLUSIONS}

In this paper, we have presented an approach, based on the embedding Green-function technique, to calculate $a b$ initio the isolated adsorption properties. We solve a selfconsistent Schrödinger equation in the density functional LDA framework in a localized region (the embedded region) containing the adsorbate and that part of the substrate mostly perturbed by it. In this approach, we take into account the effect of the solid via a nonlocal energy dependent potential defined on the surface of the embedded region (embedding potential). This potential acts as a boundary condition on the solution within the embedded region, within which the solution is unconstrained and where any suitable basis set may be used. This is different from several other treatments where the Dyson equation is used, ${ }^{17,21}$ and where the corresponding boundary conditions enter in a basis set expansion throughout the localized region.
Application of this embedding approach to an isolated $\mathrm{Si}$ adatom on jellium (Al-like) has shown the method to be capable of reproducing all the standard Lang and Williams results. ${ }^{7}$ In addition, we have made an investigation of the $\mathrm{N}$-jellium system, which exhibits bonding of primarily ionic character. The next stage in development will be to introduce a more realistic treatment of the substrate, to provide a more accurate description of real chemisorption systems.

In terms of results, perhaps the most important point of our paper is an explicit estimate of the lack of screening, both in the embedded region and in the whole system, for atomic adsorption on jellium. Our GPS method supplies a systematic evaluation of the excess of the electron charge in the whole space, and the grand-canonical energy functional provides the correction to the total energy, to first order in $\Delta Q$. Possible improvements to the problem of lack of perfect screening might involve adding an extra potential in the self-consistent equation to force global charge neutrality, as well as the obvious use of larger and larger embedded regions. This latter approach naturally becomes feasible only with greater numerical resources, but it does not solve the problem on general physical grounds.

\section{ACKNOWLEDGMENTS}

Two of us (M.I.T. and G.P.B.) are grateful to Professor Grimley for useful discussions on adsorption theory. We acknowledge useful conversations concerning embedding with J.B.A.N. van Hoof. Financial support from the E.S.F. programme "Dynamics of gas-surface interactions" is acknowledged.

\section{APPENDIX A: EMBEDDING POTENTIAL}

Consider the Green function $G\left(\mathbf{r}, \mathbf{r}^{\prime}\right)$ of a clean periodic surface and fix the $z$ direction normal to the surface, the unit cell area of which is $A$. Say $\mathbf{K}=\left(k_{x}, k_{y}\right)$ a two-dimensional reciprocal space vector, one can write ${ }^{47}$ (we drop the argument $E$ to simplify the notation)

$$
G\left(\mathbf{r}, \mathbf{r}^{\prime}\right)=\frac{A}{4 \pi^{2}} \int_{\text {First BZ }} \mathbf{d}^{2} \mathbf{K} G_{\mathbf{K}}\left(\mathbf{r}, \mathbf{r}^{\prime}\right)
$$

and using the two-dimensional Bloch properties of the wave functions, the Green function at the wave vector $\mathbf{K}$ can be expanded in a Fourier series:

$$
G_{\mathbf{K}}\left(\mathbf{R}, z ; \mathbf{R}^{\prime}, z^{\prime}\right)=\frac{1}{A} \sum_{\mu, \nu} G_{\mathbf{K}}^{\mu \nu}\left(z, z^{\prime}\right) \exp \left[i\left(\mathbf{K}_{\mu} \cdot \mathbf{R}-\mathbf{K}_{\nu} \cdot \mathbf{R}^{\prime}\right)\right]
$$

where $\mathbf{K}_{\mu}=\mathbf{K}+\mathbf{G}_{\mu}, \mathbf{R}=(x, y)$ is the component of $\mathbf{r}$ parallel to the surface and $\mathbf{G}_{\mu}$ is a surface reciprocal-lattice vector. We expand the coefficients $G_{\mathbf{K}}^{\mu \nu}\left(z, z^{\prime}\right)$ on a suitable basis set, for example, plane waves, in a region of depth $D$ in the normal direction: 


$$
G_{\mathbf{K}}^{\mu \nu}\left(z, z^{\prime}\right)=\sum_{n, n^{\prime}}^{0,1, \ldots} G_{\mathbf{K}}^{\mu \nu}\left(n, n^{\prime}\right) e^{i k_{n} z} e^{-i k_{n^{\prime}} z^{\prime}},
$$

with $k_{n}=n \pi / \tilde{D}$ and $\tilde{D}>D$. This choice is to avoid any constraints or particular boundary conditions at the limits of the region in $z$ direction.

The Green function in Eq. (A1) can now be written

$$
\begin{aligned}
G\left(\mathbf{r}, \mathbf{r}^{\prime}\right)= & \frac{1}{4 \pi^{2}} \int_{\text {First BZ }} \mathbf{d}^{2} \mathbf{K} \sum_{\mu, \nu} \sum_{n, n^{\prime}} G_{\mathbf{K}}^{\mu \nu}\left(n, n^{\prime}\right) \\
& \times \exp \left[i\left(\mathbf{K}_{\mu} \cdot \mathbf{R}+k_{n} z\right)\right] \\
& \times \exp \left[-i\left(\mathbf{K}_{\nu} \cdot \mathbf{R}^{\prime}+k_{n^{\prime}} z^{\prime}\right)\right] .
\end{aligned}
$$

To build up the embedding potential from the Green function of the clean surface, it is useful to expand the Eq. (A4) in spherical waves. Using the well known expansion,

$$
e^{i \mathbf{k} \cdot \mathbf{r}}=4 \pi \sum_{L} i^{\ell} j \ell(k r) Y_{L}^{*}\left(\Omega_{\mathbf{k}}\right) Y_{L}\left(\Omega_{\mathbf{r}}\right),
$$

and expanding the Green function as [Eq. (9)]

$$
G\left(\mathbf{r}_{S}, \mathbf{r}_{S}^{\prime}\right)=\sum_{L, L^{\prime}} \Gamma_{L L^{\prime}} Y_{L}(\Omega) Y_{L^{\prime}}^{*}\left(\Omega^{\prime}\right)
$$

we obtain the following for the matrix elements:

$$
\begin{aligned}
\Gamma_{L L^{\prime}}= & 4 i^{\ell-\ell^{\prime}} \int_{\Omega} \mathbf{d}^{2} \mathbf{K} \sum_{\mu, \nu} \sum_{n, n^{\prime}} G_{\mathbf{K}}^{\mu \nu}\left(n, n^{\prime}\right) \\
& \times j \ell\left(k_{\mu n} a\right) j_{\ell^{\prime}}\left(k_{\nu n^{\prime}} a\right) Y_{L}^{*}\left(\Omega_{k_{\mu n}}\right) Y_{L^{\prime}}\left(\Omega_{k_{\nu n^{\prime}}}\right) .
\end{aligned}
$$

Here, $k_{\mu n}=\left[\left(\mathbf{K}+\mathbf{G}_{\mu}\right)^{2}+k_{n}^{2}\right]^{1 / 2}$.

The simplest periodic substrate is the jellium surface that is invariant for any translation parallel to the surface. In this case, the only $\mathbf{G}_{\mu}$ vector that survives is $\mathbf{G}_{0}=0$, and the Brillouin zone extends to $R^{2}$. With some algebra, the matrix elements of the Green function of the jellium clean surface can be written in the following way:

$$
\begin{aligned}
\Gamma_{L L^{\prime}}= & 4 i^{\ell-\ell^{\prime}} \int_{R^{2}} \mathbf{d}^{2} \mathbf{K} \sum_{n, n^{\prime}} G_{\mathbf{K}}\left(n, n^{\prime}\right) j \curlywedge\left(k_{0 n} a\right) \\
& \times j_{\ell^{\prime}}\left(k_{0 n^{\prime}} a\right) Y_{L}^{*}\left(\Omega_{k_{0 n}}\right) Y_{L^{\prime}}\left(\Omega_{k_{0 n^{\prime}}}\right),
\end{aligned}
$$

where $k_{0 n}=\left[K^{2}+k_{n}^{2}\right]^{1 / 2}$. The matrix elements of the derivative of the Green function $\Gamma_{L L^{\prime}}^{\prime}$ [see Eq. (11)] are obtained essentially in the same way.

It is useful to stress that the Green function and its derivative exhibit a cusp and a discontinuity, respectively, when its arguments coincide $\left(\mathbf{r}=\mathbf{r}^{\prime}\right)$. This is characteristic of the embedding potential. This suggests, or better requires, us to treat analytically the singular part of the Green function in the previous equations. Note that in any numerical calculation, a finite basis set expansion is used. While a good choice of the basis can well approximate any continuous function, it cannot correctly describe a discontinuity. For this reason, we have split $G\left(\mathbf{r}, \mathbf{r}^{\prime}\right)$ into a first part $G_{\text {Sing }}\left(\mathbf{r}, \mathbf{r}^{\prime}\right)$ and a second one $G\left(\mathbf{r}, \mathbf{r}^{\prime}\right)-G_{\text {Sing }}\left(\mathbf{r}, \mathbf{r}^{\prime}\right) . G_{\text {Sing }}\left(\mathbf{r}, \mathbf{r}^{\prime}\right)$, which is known analytically, has the same singular behaviors as $G\left(\mathbf{r}, \mathbf{r}^{\prime}\right)$. The matrix elements of $G_{\text {Sing }}\left(\mathbf{r}, \mathbf{r}^{\prime}\right)$, calculated in terms of our basis set, are known analytically.

\section{APPENDIX B: ADSORPTION ENERGY}

Since the Fermi energy of the embedded system is pinned to that of the substrate, and charge is allowed to flow in and out of the embedded volume, we determine the total energy using a DF-LDA functional describing a grand-canonical ensemble. $^{38}$

$$
\begin{aligned}
E^{\mathrm{tot}}[\rho]= & \int_{-\infty}^{E_{F}} d E E \sigma(E)-\int_{R^{3}} \mathbf{d}^{3} \mathbf{r} \rho_{\mathrm{el}}(\mathbf{r}) V_{\mathrm{eff}}(\mathbf{r}) \\
& +\frac{1}{2} \int_{R^{3}} \mathbf{d}^{3} \mathbf{r} \int_{R^{3}} \mathbf{d}^{3} \mathbf{r}^{\prime} \frac{\rho(\mathbf{r}) \rho\left(\mathbf{r}^{\prime}\right)}{\left|\mathbf{r}-\mathbf{r}^{\prime}\right|} \\
& +\int_{R^{3}} \mathbf{d}^{3} \mathbf{r} \rho_{\mathrm{el}}(\mathbf{r}) \varepsilon_{\mathrm{xc}}\left(\rho_{\mathrm{el}}(\mathbf{r})\right)-E_{\mathrm{self}} \\
& -E_{F}\left(\int_{R^{3}} \mathbf{d}^{3} \mathbf{r} \rho_{\mathrm{el}}(\mathbf{r})-N\right)
\end{aligned}
$$

Apart from terms, which are found in the usual energy functionals, this includes an additional term correcting for deviations from charge neutrality. The Andersen force theorem ${ }^{39}$ may be used to show that the energy determined with this expression is correct to first order in the difference between the unperturbed density and the actual density beyond the embedding surface, and so properties obtained from the functional are less sensitive to the size of the embedded volume. $^{40}$

In our system, we treat an atom with $Z$ valence electrons, interacting with a semi-infinite jellium substrate. So that $E_{\text {self }}$ becomes

$$
E_{\text {self }}=\frac{1}{2} \int_{R^{3}} \mathbf{d}^{3} \mathbf{r} \rho_{\text {ion }}(\mathbf{r}) V_{\text {core }}(\mathbf{r}),
$$

where $\rho_{\text {ion }}(\mathbf{r})=Z \delta\left(\mathbf{r}-\mathbf{R}_{a}\right)$ is the positive charge of the pseudoatom and $V_{\text {core }}(\mathbf{r})$ is defined in Eqs. (19) and (20).

We are interested in the interaction energy, i.e., the difference between the energy of the whole system, surface + adatom, and the energies of both isolated system, i.e., that of clean surface $E^{0}$ and that of the isolated atom $E^{\text {at }}$. Within the hypothesis that the potentials and the charge distribution outside the embedding sphere are unaffected with respect to the unperturbed situation, we obtain the following interaction energy: 


$$
\begin{aligned}
\mathscr{E}=E^{\mathrm{tot}}-E^{0}-E^{\mathrm{at}}= & \left\{\int_{-\infty}^{E_{F}} d E E \sigma(E)-\int_{I} \mathbf{d}^{3} \mathbf{r} \rho_{\mathrm{el}}(\mathbf{r}) V_{\mathrm{eff}}(\mathbf{r})+\frac{1}{2} \int_{I} \mathbf{d}^{3} \mathbf{r} \int_{R^{3}} \mathbf{d}^{3} \mathbf{r}^{\prime} \frac{\rho(\mathbf{r}) \rho\left(\mathbf{r}^{\prime}\right)}{\left|\mathbf{r}-\mathbf{r}^{\prime}\right|}+\int_{I} \mathbf{d}^{3} \mathbf{r} \rho_{\mathrm{el}}(\mathbf{r}) \varepsilon_{\mathrm{xc}}\left(\rho_{\mathrm{el}}(\mathbf{r})\right)-E_{\mathrm{self}}\right\} \\
& -\left\{\int_{-\infty}^{E_{F}} d E E \sigma^{0}(E)-\int_{I} \mathbf{d}^{3} \mathbf{r} \rho_{\mathrm{el}}^{0}(\mathbf{r}) V_{\mathrm{eff}}^{0}(\mathbf{r})+\frac{1}{2} \int_{I} \mathbf{d}^{3} \mathbf{r} \int_{R^{3}} \mathbf{d}^{3} \mathbf{r}^{\prime} \frac{\rho^{0}(\mathbf{r}) \rho^{0}\left(\mathbf{r}^{\prime}\right)}{\left|\mathbf{r}-\mathbf{r}^{\prime}\right|}+\int_{I} \mathbf{d}^{3} \mathbf{r} \rho_{\mathrm{el}}^{0}(\mathbf{r}) \varepsilon_{\mathrm{xc}}\left(\rho_{e l}^{0}(\mathbf{r})\right)\right\} \\
& -E_{F}\left(\int_{R^{3}} \mathbf{d}^{3} \mathbf{r}\left[\rho_{\mathrm{el}}(\mathbf{r})-\rho_{\mathrm{el}}^{0}(\mathbf{r})\right]-Z\right)-E^{\mathrm{at}} .
\end{aligned}
$$

All quantities with the superscript 0 refer to the clean surface. The atomic energy $E^{\text {at }}$ can be determinated by a standard procedure in the embedding region. As mentioned in the text, the integrals involving the density of states $\sigma(E)$ (the band structure part) need more attention, because the individual states change throughout the whole system:

$$
\int_{-\infty}^{E_{F}} d E E\left[\sigma(E)-\sigma^{0}(E)\right]=\int_{-\infty}^{E_{F}} d E E \Delta \sigma(E)=\left[E \int_{-\infty}^{E} d \epsilon \Delta \sigma(\epsilon)\right]_{-\infty}^{E_{F}}-\int_{-\infty}^{E_{F}} d E \int_{-\infty}^{E} d \epsilon \Delta \sigma(\epsilon),
$$

and using the definition in Eqs. (28),

$$
=E_{F} \Delta N\left(E_{F}\right)-\int_{-\infty}^{E_{F}} d E \Delta N(E)
$$

The last integral in Eq. (B3) can be transformed, using the definitions in Sec. III:

$$
\int_{R^{3}} \mathbf{d}^{3} \mathbf{r}\left[\rho_{\mathrm{el}}(\mathbf{r})-\rho_{\mathrm{el}}^{0}(\mathbf{r})\right]=\int_{-\infty}^{E_{F}} d E \Delta \sigma(E)=\Delta N\left(E_{F}\right) .
$$

Finally, the interaction energy can be written in the following way:

$$
\begin{aligned}
\mathscr{E}= & E_{F} Z-\int_{-\infty}^{E_{F}} d E \Delta N(E)-\int_{I} \mathbf{d}^{3} \mathbf{r} \rho_{\mathrm{el}}(\mathbf{r}) V_{\mathrm{eff}}(\mathbf{r})+\frac{1}{2} \int_{I} \mathbf{d}^{3} \mathbf{r} \int_{R^{3}} \mathbf{d}^{3} \mathbf{r}^{\prime} \frac{\rho(\mathbf{r}) \rho\left(\mathbf{r}^{\prime}\right)}{\left|\mathbf{r}-\mathbf{r}^{\prime}\right|}+\int_{I} \mathbf{d}^{3} \mathbf{r} \rho_{\mathrm{el}}(\mathbf{r}) \varepsilon_{\mathrm{xc}}\left(\rho_{\mathrm{el}}(\mathbf{r})\right)-E_{\mathrm{self}} \\
& +\int_{I} \mathbf{d}^{3} \mathbf{r} \rho_{\mathrm{el}}^{0}(\mathbf{r}) V_{\mathrm{eff}}^{0}(\mathbf{r})-\frac{1}{2} \int_{I} \mathbf{d}^{3} \mathbf{r} \int_{R^{3}} \mathbf{d}^{3} \mathbf{r}^{\prime} \frac{\rho^{0}(\mathbf{r}) \rho^{0}\left(\mathbf{r}^{\prime}\right)}{\left|\mathbf{r}-\mathbf{r}^{\prime}\right|}-\int_{I} \mathbf{d}^{3} \mathbf{r} \rho_{\mathrm{el}}^{0}(\mathbf{r}) \varepsilon_{\mathrm{xc}}\left(\rho_{\mathrm{el}}^{0}(\mathbf{r})\right)-E^{\mathrm{at}}
\end{aligned}
$$

*Present address: Department of Physics and Astronomy, University of Wales College of Cardiff, P.O. Box 913, Cardiff CF2 3YB, UK.

${ }^{1}$ G.P. Brivio and T.B. Grimley, Surf. Sci. Rep. 17, 1 (1993).

${ }^{2}$ B.I. Lundqvist, in Interaction of Atom and Molecules with Solid Surfaces, edited by V. Bortolani, N.H. March, and M.P. Tosi (Plenum Press, New York, 1990), p. 213.

${ }^{3}$ T.B. Grimley, in The Nature of the Surface Chemical Bond, edited by T.N. Rhodin and G. Ertl (North-Holland, Amsterdam, 1979), p. 1.

${ }^{4}$ T.B. Grimley and C. Pisani, J. Phys. C 7, 2831 (1974).

${ }^{5}$ O. Gunnarsson, H. Hjelmberg, and B.I. Lundqvist, Phys. Rev. Lett. 37, 292 (1976).

${ }^{6}$ O. Gunnarsson and H. Hjelmberg, Phys. Scr. 11, 97 (1975).

${ }^{7}$ N.D. Lang and A.R. Williams, Phys. Rev. B 18, 616 (1978).

${ }^{8}$ P. Hohenberg and W. Kohn, Phys. Rev. 136, B864 (1964).

${ }^{9}$ W. Kohn and L.J. Sham, Phys. Rev. 140, A1133 (1965).

${ }^{10}$ S. Lundqvist and N.H. March, Theory of the Inhomogeneous Electron Gas (Plenum Press, New York, 1983).

${ }^{11}$ J.P. Perdew, in Electronic Structure of Solids, edited by P. Ziesche and H. Eschrig (Akademie Verlag, Berlin, 1991).

${ }^{12}$ For example, see Cluster Models for Surfaces and Bulk Phenomena, Vol. 283 of NATO Advanced Study Institute, Series B: Physics, edited by G. Pacchioni, P.S. Bagus, and F. Parmigiani (Plenum, New York, 1992); Metal-Ligand Interactions: From Atoms to Clusters to Surfaces, Vol. 378 of NATO Advanced Study Institute, Series C: Mathematical and Physical Sciences, edited by D.R. Salahub and N. Russo (Kluwer, Dordrecht, 1992).

${ }^{13}$ J. Hebenstreit and M. Scheffler, Phys. Rev. B 46, 10134 (1992).

${ }^{14}$ D.M. Bird, L.J. Clarke, M.C. Payne, and I. Stich, Chem. Phys. Lett. 212, 518 (1993).

${ }^{15}$ A preliminary report of part of this work has been previously presented (Ref. 16), where results for the charge density of a $\mathrm{Si}$ atom on $\mathrm{Al}$ (jellium) were given at a single atom-surface separation. The present account extends this work, providing a fuller account of the method and reporting the adsorption energetics and electronic properties of both $\mathrm{Si}$ and $\mathrm{N}$ on $\mathrm{Al}$ (jellium) over a wide range of atom-surface separations. There are also some technical differences in the calculations, such as basis functions and calculation parameters-since the atom is now also considered at off-center positions, a larger sphere radius is required and this forces a larger angular momentum cutoff.

${ }^{16}$ M.I. Trioni, J.B.A.N. van Hoof, S. Crampin, G.P. Brivio, and J.E. Inglesfield, Surf. Sci. 307-309, 41 (1994).

${ }^{17}$ M. Scheffler, Ch. Droste, A. Fleszar, F. Máca, G. Wachutka, and G. Borzel, Physica B 172, 143 (1991).

${ }^{18}$ J. Bormet, J. Neugebauer, and M. Scheffler, Phys. Rev. B 49, 17242 (1994).

${ }^{19}$ P. Lang, V.S. Stepanyuk, K. Wildberger, R. Zeller, and P.H. Dederichs, Solid State Commun. 92, 755 (1994). 
${ }^{20}$ M. Aldén, I.A. Abrikosov, B. Johansson, N.M. Rosengaard, and H.L. Skriver, Phys. Rev. B 50, 5131 (1994).

${ }^{21}$ P.J. Feibelman, Phys. Rev. B 35, 2626 (1989).

${ }^{22}$ P. Nordlander and J.C. Tully, Phys. Rev. B 42, 5564 (1990).

${ }^{23}$ J.E. Inglesfield, J. Phys. C 14, 3795 (1981).

${ }^{24}$ G.A. Benesh and J.E. Inglesfield, J. Phys. C 17, 1595 (1984).

${ }^{25}$ J.E. Inglesfield and G.A. Benesh, Phys. Rev. B 37, 6682 (1988).

${ }^{26}$ M. Nekovee, S. Crampin, and J.E. Inglesfield, Phys. Rev. Lett. 70, 3099 (1993).

${ }^{27}$ S. Crampin, M.H. Boon, and J.E. Inglesfield, Phys. Rev. Lett. 73, 1015 (1994).

${ }^{28}$ G.A. Benesh and L.S.G. Liyanage, Phys. Rev. B 49, 17264 (1994).

${ }^{29}$ J.B.A.N. van Hoof, S. Crampin, and J.E. Inglesfield, J. Phys. Condens. Matter 4, 8477 (1992).

${ }^{30}$ S. Crampin, M. Nekovee, J.B.A.N van Hoof, and J.E. Inglesfield, Surf. Sci. 287/288, 732 (1993).

${ }^{31}$ S. Crampin, J.B.A.N. van Hoof, M. Nekovee, and J.E. Inglesfield, J. Phys. Condens. Matter 4, 1475 (1992).

${ }^{32}$ F. García-Moliner and J. Rubio, J. Phys. C 2, 1789 (1969); J.E. Inglesfield, ibid. 4, L14 (1971).

${ }^{33}$ G. Arfken, Mathematical Methods for Physicists (Academic Press, New York, 1985).

${ }^{34}$ J.B. Pendry, Low-Energy Electron Diffraction (Academic Press, New York, 1974).
${ }^{35}$ G.B. Bachelet, D.R. Hamann, and M. Schlüter, Phys. Rev. B 26, 4199 (1982).

${ }^{36}$ Of course, deeper lying core levels, which exhibit no dispersion, are best treated like atomic levels, rather than by extending the energy contour to encompass them. Here, we implement the method with pseudopotentials, which do not possess deep levels, and for which all states may be included within the contour.

${ }^{37}$ J.E. Inglesfield and G.A. Benesh, Surf. Sci. 200, 135 (1988).

${ }^{38}$ N.D. Mermin, Phys. Rev. 137, A1441 (1965).

${ }^{39}$ A.R. Mackintosh and O.K. Andersen, in Electrons at the Fermi Surface, edited by M. Springford (Cambridge University Press, London, 1980).

${ }^{40}$ B. Drittler, M. Weinert, R. Zeller, and P.H. Dederichs, Phys. Rev. B 39, 930 (1989).

${ }^{41}$ There is a discrepancy in Eq. (29) of the present treatment and Eq. (18) in Ref. 18 in the meaning of $\Delta Q$.

${ }^{42} a_{0}$ is the Bohr radius.

${ }^{43}$ D.M. Ceperly and B.J. Alder, Phys. Rev. Lett. 45, 566 (1980).

${ }^{44}$ M. Okada and Y. Murata, J. Phys. Condens. Matter 4, 5097 (1992).

${ }^{45}$ J.A. Taylor and J.W. Rabalais, J. Chem. Phys. 75, 1735 (1981).

${ }^{46}$ P.J. Rous, in Cohesion and Structure Vol. IV, Cohesion and Structure of Surfaces, edited by D.G. Pettifor (North-Holland, Amsterdam, 1995).

${ }^{47}$ J.E. Inglesfield, Surf Sci. 76, 355 (1978). 